\title{
genieR: An R package for inference of demographic history of phylogenies
}

\author{
Fei Xiang ${ }^{1}$, Bethany Dearlove ${ }^{2}$, and Simon Frost ${ }^{1}$ \\ 1 Department of Veterinary Medicine, University of Cambridge, Cambridge, United Kingdom 2 US \\ Military HIV Research Program, Rockville, MD, United States
}

DOI: $10.21105 /$ joss.00634

\section{Software}

- Review ¿

- Repository $\iota^{\top}$

- Archive ct

Submitted: 16 January 2018

Published: 05 January 2019

\section{License}

Authors of papers retain copyright and release the work under a Creative Commons Attribution 4.0 International License (CC-BY).

\section{Summary}

genieR is an $\mathrm{R}$ package ( $\mathrm{R}$ Core Team (2016)) for the inference of demographic history from reconstructed molecular phylogenies (see Dearlove, Xiang, \& Frost (2017) for one application), modeled after the $\mathrm{C}++$ package GENIE (Pybus \& Rambaut, 2002). The package processes phylogenetic trees in Newick format, in which taxa may be sampled either at the same time (isochronous) or at different times (heterochronous); the latter are particularly common in the analysis of pathogen sequence data. In addition to extracting basic information from a particular phylogeny such as sampling and coalescent times, genieR also fits a coalescent model (Kingman (1982)) under several demographic scenarios, including constant population size, exponential growth, expansion growth, logistic growth, with both continuous and piecewise variants. The fit of different models can be compared using Akaike's Information Criterion (AIC) (Akaike, 1973) values. genieR uses C++ code as an option for better computational performance, and models can be fit either using maximum likelihood or Markov Chain Monte Carlo (Hastings, 1970). genieR can also simulate phylogenies under different demographic models.

\section{References}

Akaike, H. (1973). Information theory and an extension of the maximum likelihood principle. In Second international symposium on information theory (pp. 267-281). Academiai Kiado: Budapest.

Dearlove, B. L., Xiang, F., \& Frost, S. D. W. (2017). Biased phylodynamic inferences from analysing clusters of viral sequences. Virus Evolution, 3(2). doi:10.1093/ve/vex020

Hastings, W. K. (1970). Monte carlo sampling methods using markov chains and their applications. Biometrika, 57(1), 97-109. doi:10.2307/2334940

Kingman, J. F. C. (1982). On the genealogy of large populations. Journal of Applied Probability, 19, 27-43. doi:10.2307/3213548

Pybus, O. G., \& Rambaut, A. (2002). GENIE: Estimating demographic history from molecular phylogenies. Bioinformatics, 18, 1404-1405. doi:10.1093/bioinformatics/18.10.1404

R Core Team. (2016). R: A language and environment for statistical computing. Vienna, Austria: R Foundation for Statistical Computing. Retrieved from http://www.R-project. org/ 